\title{
Topological order and the deconfinement transition in the $(2+1)$ dimensional compact Abelian Higgs model
}

\author{
Anders Vestergren $*$ and Jack Lidmali \\ Department of Physics, Royal Institute of Technology, AlbaNova, SE-106 91 Stockholm, Sweden
}

(Dated: November 20, 2018)

\begin{abstract}
We study an Abelian compact gauge theory minimally coupled to bosonic matter with charge $q$, which may undergo a confinement-deconfinement transition in $(2+1) \mathrm{D}$. The transition is analyzed using a nonlocal order parameter $\tilde{W}$, which is related to large Wilson loops for fractional charges. We map the model to a dual representation with no gauge field but only a global $q$-state clock symmetry and show that $\tilde{W}$ correspond to the domain wall energy of that model. $\tilde{W}$ is also directly connected to the concept of topological order. We exploit these facts in Monte Carlo simulations to study the detailed nature of the deconfinement transition.

PACS numbers: $74.20 .-\mathrm{z}, 11.15 . \mathrm{Ha}, 71.10 . \mathrm{Hf}$
\end{abstract}

\section{INTRODUCTION}

The appearance of excitations with fractional quantum numbers in strongly correlated systems has received

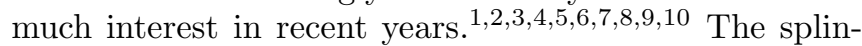
tering of the electron into a neutral spinon and a charged spinless holon, and the resulting spin-charge separation, was proposed early after the discovery of the superconducting high- $T_{c}$ cuprates as a route to explain the nonFermi liquid behavior observed in these systems. The fractionally charged Laughlin quasi-particles in the fractional quantum hall effect is another famous example. While spin-charge separation is well established in one dimensional electron gases, the occurrence in higher dimensions remains poorly understood. Usually, the fractionalization in dimensions larger than one, is imagined to occur via a confinement-deconfinement transition of an effective low energy gauge theory description of the system 11.12 A number of such gauge theories have been proposed for the underdoped cuprates, including

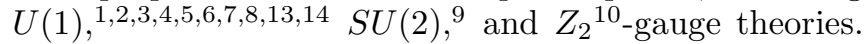
In addition, the possibility of fractionalized phases in models containing only bosons has been proposed 15 The fractionalized phases are not ordered in a conventional sense, which breaks any obvious symmetries of the system. Instead they are characterized by a special kind of order called topological order ${ }^{16}$ Topological order is robust against local perturbations and furthermore leads to a ground state degeneracy. It has been proposed that this topologically protected degeneracy could serve as a building block for qubits in a quantum computer ${ }^{17.18} \mathrm{An}$ important prediction is that in finite sized systems the degeneracy is lifted due to tunneling processes, leading to a splitting $\Delta \sim e^{-L / \xi}, \underline{16}$

In this paper we study the relation between topological order and deconfinement in one of the simplest models displaying topological order - the compact Abelian Higgs model. It should be noted that a realistic theory of the high- $T_{c}$ cuprates would require also the inclusion of gapless nodal quasiparticles, which are here neglected $19,20.21 .22 .23$ We thus consider a $(2+1) \mathrm{D}$ com- pact $U(1)$ gauge theory minimally coupled to a charge- $q$ bosonic matter field, with an Euclidean action

$$
S=-J \sum_{\mathbf{r} \mu} \cos \left(\nabla_{\mu} \theta_{\mathbf{r}}-q A_{\mathbf{r} \mu}\right)-\frac{1}{g} \sum_{\mathbf{r} \mu} \cos \left(B_{\mathbf{r} \mu}\right),
$$

where $\theta_{\mathbf{r}}$ and $A_{\mathbf{r} \mu}$ are compact phases $\in[0,2 \pi)$ living on the sites and links of a $3 \mathrm{D}$ simple cubic lattice, respectively. $B_{\mathbf{r} \mu}=\epsilon_{\mu \nu \lambda} \nabla_{\nu} A_{\mathbf{r} \lambda}$ is the dual field strength, with the lattice difference operators defined by $\nabla_{\mu} f_{\mathbf{r}}=\bar{\nabla}_{\mu} f_{\mathbf{r}+\mathbf{e}_{\mu}}=f_{\mathbf{r}+\mathbf{e}_{\mu}}-f_{\mathbf{r}}$. The pure gauge theory in absence of matter is always confining in $(2+1) \mathrm{D}$, due to the proliferation of instantons ${ }^{24}(\operatorname{In}(3+1) \mathrm{D}$ a deconfined Coulomb phase is also possible. ${ }^{24}$ Partly because of this much attention has focused on $Z_{2}$-gauge theories $\stackrel{10}{\underline{\underline{n}}}$ which do allow a confinement-deconfinement transition in $(2+1) \mathrm{D}$. Alternatively, when the $U(1)$ model is coupled to a matter field which does not belong to the fundamental representation (i.e., when the charge $q$ of the boson differs from unity in Eq. (1)), a phase transition is possible ${ }^{25}$ This could happen, e.g., if the bosonic field describes the pairing of spinons $\frac{5,26}{\text { Indeed, the } U(1)}$ gauge theory coupled to a charge 2 boson is equivalent to a $Z_{2}$-gauge theory in the limit $J \rightarrow \infty$. In such a system, the fractionalized phase would then correspond to a phase where the spinon-pairs breaks up into separate free excitations. More generally, the model with gauge charge $q$ reduces to a $Z_{q}$-gauge theory in the limit $J \rightarrow \infty$. In the opposite limit $g \rightarrow 0$, the gauge field fluctuations freeze out and a 3D $X Y$ model (with a global $\mathrm{U}(1)$ symmetry) results. One may ask how the transition interpolates between these two extremes with increasing screening length $\lambda=1 / \sqrt{J g q^{2}}$. The naive expectation would be that the $\lambda \rightarrow \infty$ transition would represent an unstable fixed point and that any finite value of $\lambda$ would flow to zero upon renormalization, giving a transition in the $Z_{q}$ universality class. In this case the $3 \mathrm{D} X Y$ point at $\lambda \rightarrow \infty$ would be an isolated point in the phase diagram.

Recently, large scale Monte Carlo simulations of the $(2+1) \mathrm{D}$ compact $U(1)$ Higgs model were performed by Sudb $\varnothing$ et $a l^{27}$ and Smiseth et al. 28 for several values of the charge $q>1$. The results of these indicated that the 
phase diagram might be surprisingly complicated, with continuously varying critical exponents along the transition line between the $Z_{q}$ and 3D $X Y$ values. This would imply that the transition line for intermediate $\lambda$ might be described by a line of fixed points. These interesting results were obtained from a finite size scaling analysis of the third moment of the action, which is a purely thermodynamic observable. Although their method seems to work very well, it would be desirable to have a clear order parameter which distinguishes the confining and deconfining phases, especially in light of their surprising results.

In this paper we employ a nonlocal order parameter $\tilde{W}$, previously introduced in Ref 29 , which provides a direct probe of the confining properties of the theory. We show that this order parameter can be mapped via a duality transformation to the domain wall energy of a $Z_{q}$ clock model, and present an in depth discussion of its relation to topological order, including many of the details left out in Ref 29. We obtain the finite size scaling properties of $\tilde{W}$, discuss its relation to the splitting $\Delta$ of the nearly degenerate ground states, and the energy gap to vortex excitations in the deconfined phase. Finally, we use it in Monte Carlo simulations to study the detailed nature of the deconfinement transition of the model, and compare with the earlier results mentioned above.

The paper is organized as follows. In Sec. IIA we reformulate the theory in terms of its topological defects, which are vortices and instantons, and discuss how $\tilde{W}$ can distinguish the different phases. Going over to another dual formulation a model with a global $q$-state clock symmetry (and no gauge fields) obtains, and $\tilde{W}$ translates into the domain wall energy of that model, or equivalently the energy cost for twisting the boundary conditions by $2 \pi / q$, see Sec. IIB IIC In Sec. IID we discuss the relation to topological order, adding details and generalizations of the ideas discussed in Ref 29. In Sec.III) we describe Monte Carlo simulations of the $(2+1) \mathrm{D}$ compact Higgs model carried out in the loop-gas representation of flux lines and monopoles. Our results and conclusions are discussed in Sec. [IV] and $\mathrm{D}$.

\section{DUALITY TRANSFORMATIONS, WILSON LOOPS AND TOPOLOGICAL ORDER}

In order to transform Eq. (1) to alternative representations it is convenient to instead study the Villain version of the model, which allow the action to be transformed to dual representations exactly, without further approximations, while retaining the important physics. In the Villain approximation the action is ing the energy cost of putting an integer multiple of $q$ flux quanta $\Phi_{0}=2 \pi / q$ through a plaquette of the lattice vanish. The net effect of this is the proliferation of Dirac strings, which leads to the appearance of magnetic monopoles of charge $q \Phi_{0}(=2 \pi)$.

A standard set of transformations ${ }^{25.30}$ allows Eq. (2) to be rewritten in terms of flux lines and monopoles, interacting via the action

$$
S=\sum_{\mathbf{r} \mathbf{r}^{\prime}} \frac{K}{2} \mathbf{m}_{\mathbf{r}} \cdot V_{\mathbf{r} \mathbf{r}^{\prime}} \mathbf{m}_{\mathbf{r}^{\prime}}+\frac{K \lambda^{2} q^{2}}{2} N_{\mathbf{r}} V_{\mathbf{r r}^{\prime}} N_{\mathbf{r}^{\prime}}
$$

Here $\mathbf{m}_{\mathbf{r}} \in \mathbb{Z}^{3}$ is the vorticity on the links and $N_{\mathbf{r}} \in \mathbb{Z}$ is the monopole charge, ${ }^{36}$ and $K=(2 \pi)^{2} J$. The flux lines are constrained to start or end on the monopoles only in quanta of $q$, i.e., $\nabla \cdot \mathbf{m}_{\mathbf{r}}=q N_{\mathbf{r}}$. The flux lines and monopoles interact through a screened coulomb interaction, defined by its Fourier transform $V_{\mathbf{k}}^{-1}=\kappa_{\mathbf{k}}^{2}+\lambda^{-2}$, where $\kappa^{2}=\sum_{\nu} \kappa_{\mathbf{k} \nu}^{2}$, and $\kappa_{\mathbf{k} \nu}=2 \sin \left(k_{\nu} / 2\right)$, i.e.,

$$
V_{\mathbf{r}}=\frac{1}{L^{3}} \sum_{\mathbf{k}} \frac{e^{i \mathbf{k} \cdot \mathbf{r}}}{\kappa_{\mathbf{k}}^{2}+\lambda^{-2}}
$$

which for large distances is a screened coulomb interaction $V(r)=e^{-r / \lambda} / 4 \pi r$ with a screening length $\lambda$ given by $\lambda^{-2}=K g / \Phi_{0}^{2}=J g q^{2}$. In the limit $\lambda \rightarrow 0$ the interaction reduces to an onsite interaction $V_{\mathbf{r}}=\lambda^{2} \delta_{\mathbf{r}}$, and the action becomes

$$
S=\frac{\Phi_{0}^{2}}{2 g} \sum_{\mathbf{r}} \mathbf{m}_{\mathbf{r}}^{2}
$$

The constraint $\nabla \cdot \mathbf{m}=q N$ means that a $+(-)$ monopole has precisely $q$ outgoing (incoming) flux lines. For $q>1$ one may then envisage two distinct phases of the system. Either the monopoles mostly pair up in neutral pairs, where the $q$ outgoing flux lines of the plus charge all end at the minus charge of the pair, as illustrated in Fig. 1(a). This phase will be realized in the limit of large $K$, where the flux lines cost a lot of energy. As discussed in the next section, this magnetically confining phase corresponds to deconfined fractional electric charges. The other possibility is that the outgoing flux lines mostly end on different monopoles, forming a large connected tangle of flux lines and monopoles, which percolate through the whole system, as in Fig. 1(b). Recent simulations ${ }^{31}$ support this interpretation. A transition between these two geometrically different phases is expected at some critical value of the coupling $K_{c}$ (or $g_{c}$ ). This picture can be made more precise by studying the Wilson loop for fractional charges.

\section{A. Wilson loops}

The presence of percolating flux lines in the system can be detected by counting the number of flux lines crossing a given surface $\mathcal{S}$ that form a cross-section of the system, where $n_{\mathbf{r} \mu}$ and $k_{\mathbf{r} \mu}$ are dummy integers to be summed over. The summation over $k_{\mathbf{r} \mu}$ has the effect of mak-
$S=\sum_{\mathbf{r} \mu} \frac{J}{2}\left(\nabla_{\mu} \theta_{\mathbf{r}}+2 \pi n_{\mathbf{r} \mu}-q A_{\mathbf{r} \mu}\right)^{2}+\sum_{\mathbf{r} \mu} \frac{1}{2 g}\left(B_{\mathbf{r} \mu}+2 \pi k_{\mathbf{r} \mu}\right)^{2}$, 

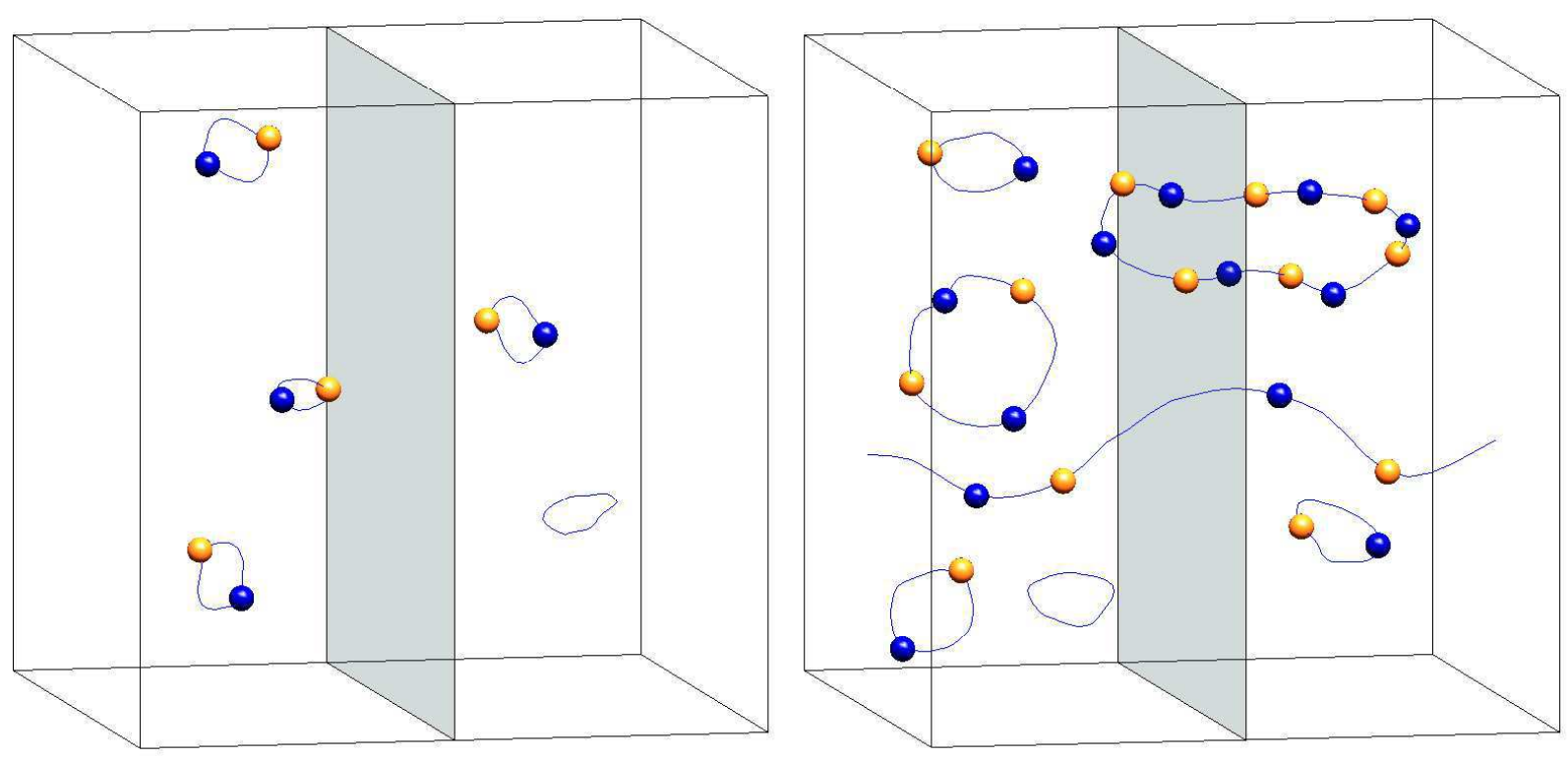

FIG. 1: (Color online) Possible phases of the system. To the left the topological ordered, deconfined phase, where vortices cost much energy. To the right the percolating vortex tangle in the confined phase. The large Wilson loop $\tilde{W}$ surrounds the shaded area as indicated in the figures.

see Fig. 1. We assume here periodic boundary conditions for the vorticity $\mathbf{m}$ in all directions, i.e., the system has the topology of a 3-torus. This corresponds to allowing twists in the boundary conditions for A in Eq. (11) or (21) ${ }^{29}$ Let the winding number $M_{\nu}$ be the number of such flux line crossings (counted with signs) for a cross-section perpendicular to the $\nu$-direction, i.e.,

$$
M_{\nu}=\int_{\mathcal{S}} \mathbf{m} \cdot d \mathbf{S}
$$

(For notational convenience we use a continuum formulation here.) The precise location of the surface does not matter. In the paired phase this number will be an integer multiple of $q$, whereas in the percolating phase it can be any integer. It turns out that precisely this property is captured by a large Wilson loop for fractionally charged test particles,

$$
\tilde{W} \equiv W(\mathcal{C})=\left\langle\exp \left(i \oint_{\mathcal{C}} \mathbf{A} \cdot d \mathbf{r}\right)\right\rangle,
$$

where the loop $\mathcal{C}$ encircles the surface $\mathcal{S}$. The expression in the exponent,

$$
\oint_{\mathcal{C}} \mathbf{A} \cdot d \mathbf{r}=\int_{\mathcal{S}} \mathbf{B} \cdot d \mathbf{S}=\frac{2 \pi}{q} \int_{\mathcal{S}} \mathbf{m} \cdot d \mathbf{S}
$$

equals $2 \pi / q$ times the winding number $M_{\nu}$, and the Wilson loop for a loop with this special geometry is thus given by

$$
\tilde{W}=\left\langle\exp \left(\frac{2 \pi i}{q} M_{\nu}\right)\right\rangle .
$$

This becomes one in the paired phase where $M_{\nu}$ is an integer multiple of $q$ [Fig. 1(a)]. In the percolating phase [Fig. [(b)] no particular value of the winding number $M_{\nu}$ will be favored, making the $\tilde{W}$ approach zero with increasing system size. Note that it is the global topological structure of the flux line tangle that determines the value of $\tilde{W}$, while small scale entanglement is unimportant.

A Wilson loop decaying with an area law, $W(\mathcal{C}) \sim$ $\exp \left(-c L^{2}\right)$ implies a linearly confining attraction between static fractional charges, while a perimeter law, $W(\mathcal{C}) \sim \exp (-c L)$, means that such charges would be deconfined. Note that the behavior of the charges is opposite of the monopoles: The charges are deconfined when the monopoles form pairs and confined when the pairs break up. Remember that the charge of the bosons in the original model is $q$, so the Wilson loop for charge- $q$ test particles would include a factor $q$ in the exponent, making $\tilde{W}$ equal to 1 exactly in both phases. For fractional charges, however, it changes from unity to an area law $\tilde{W} \sim \exp \left(-c L^{2}\right)$, when going through the transition (deep in the confined phase one finds $c=g / 2$ ). Another way to say this is that the charge- $q$ dynamic matter cannot screen out the fractionally charged test particles.

The absence of a perimeter law for $\tilde{W}$ in the deconfined phase is a consequence of the special geometry of a loop which covers a whole cross-section of the system, and in some sense has no perimeter due to the periodic boundary conditions. This makes our order parameter $\tilde{W}$ different from ordinary Wilson loops, and is a big advantage since it makes it easier to analyze its scaling 
behavior as discussed in Sec. IIC For a finite sized loop in a much larger system the Wilson loop would follow a perimeter law rather than approach unity in the deconfined phase. This is due to configurations where part of the flux connecting a monopole-anti-monopole pair would pass through the loop and part outside the loop. In the paired phase this can happen only very close to the loop itself, hence giving rise to a perimeter law. In the next two sections we describe the relation of the Wilson loop order parameter $\tilde{W}$ to the domain wall energy in a dual clock-symmetric model.

\section{B. Mapping to a model with clock symmetry}

We will now map Eq. (3) to a dual model with a $q$-state clock symmetry. The idea is to start from the action

$$
\begin{aligned}
S & =\sum_{\mathbf{r r}^{\prime}} \frac{K}{2} \mathbf{m}_{\mathbf{r}} \cdot V_{\mathbf{r r}^{\prime}} \mathbf{m}_{\mathbf{r}^{\prime}}+\frac{K \lambda^{2} q^{2}}{2} N_{\mathbf{r}} V_{\mathbf{r r}^{\prime}} N_{\mathbf{r}^{\prime}} \\
& +\sum_{\mathbf{r}} i \chi_{\mathbf{r}}\left(\nabla \cdot \mathbf{m}_{\mathbf{r}}-q N_{\mathbf{r}}\right)
\end{aligned}
$$

where the constraint $\nabla \cdot \mathbf{m}=q N$ is implemented via a Lagrange multiplier $\chi_{\mathbf{r}}$, and then integrate out the flux lines and monopoles to get an effective action for $\chi$. We decouple the vortex-vortex and monopole-monopole interactions with the help of two auxiliary fields, a and $\phi$, to get

$$
\begin{aligned}
S & =\sum_{\mathbf{r \mathbf { r } ^ { \prime }}} \frac{1}{2 K} \mathbf{a}_{\mathbf{r}} \cdot V_{\mathbf{r r}^{\prime}}^{-1} \mathbf{a}_{\mathbf{r}^{\prime}}+\frac{1}{2 K \lambda^{2}} \phi_{\mathbf{r}} V_{\mathbf{r r}^{\prime}}^{-1} \phi_{\mathbf{r}^{\prime}} \\
& +\sum_{\mathbf{r}} i \mathbf{a}_{\mathbf{r}} \cdot \mathbf{m}_{\mathbf{r}}+i \phi_{\mathbf{r}} q N_{\mathbf{r}}+i \chi_{\mathbf{r}}\left(\nabla \cdot \mathbf{m}_{\mathbf{r}}-q N_{\mathbf{r}}\right)(11)
\end{aligned}
$$

Now the summation over the integers $\mathbf{m}_{\mathbf{r}}$ and $N_{\mathbf{r}}$ can be performed, leading to

$$
\mathbf{a}=\nabla \chi+2 \pi \mathbf{n}, \quad \phi=\chi+2 \pi k / q, \quad \mathbf{n}, k \text { integers. }
$$

We then obtain the action

$$
\begin{aligned}
S & =\sum_{\mathbf{r} \mathbf{r}^{\prime}} \frac{1}{2 K}\left(\nabla \chi_{\mathbf{r}}+2 \pi \mathbf{n}_{\mathbf{r}}\right) V_{\mathbf{r r}^{\prime}}^{-1}\left(\nabla \chi_{\mathbf{r}^{\prime}}+2 \pi \mathbf{n}_{\mathbf{r}^{\prime}}\right) \\
& +\frac{1}{2 K \lambda^{2}}\left(\chi_{\mathbf{r}}+2 \pi k_{\mathbf{r}} / q\right) V_{\mathbf{r r}^{\prime}}^{-1}\left(\chi_{\mathbf{r}^{\prime}}+2 \pi k_{\mathbf{r}^{\prime}} / q\right) .
\end{aligned}
$$

As announced this model has only a global $q$-state clock symmetry and contains no gauge fields and only short range interactions. Rewriting it in Fourier space gives

$$
\begin{aligned}
S & =\frac{1}{2 K \Omega} \sum_{\mathbf{k}}\left(\kappa^{2}+\lambda^{-2}\right)\left|\boldsymbol{\kappa} \chi_{\mathbf{k}}+2 \pi \mathbf{n}_{\mathbf{k}}\right|^{2} \\
& +\frac{1}{\lambda^{2}}\left(\kappa^{2}+\lambda^{-2}\right)\left|\chi_{\mathbf{k}}+2 \pi k_{\mathbf{k}} / q\right|^{2}
\end{aligned}
$$

Obviously $V_{\mathbf{k}}^{-1}=\kappa^{2}+\lambda^{-2}$ is short ranged and may at least for small values of $\lambda$ (i.e. large $g$ ) be replaced by a completely local interaction, $V_{\mathbf{r r}^{\prime}}^{-1} \approx \lambda^{-2} \delta_{\mathbf{r r}^{\prime}}$. The corrections enter with higher order derivative and should therefore be irrelevant at small $\lambda$. Dropping these and "undoing" the Villain approximation now gives

$$
S \approx-\frac{1}{K \lambda^{2}}\left\{\sum_{\mathbf{r} \mu} \cos \left(\nabla_{\mu} \chi_{\mathbf{r}}\right)+\frac{1}{q^{2} \lambda^{2}} \sum_{\mathbf{r}} \cos \left(q \chi_{\mathbf{r}}\right)\right\}
$$

which clearly has a $q$-state clock symmetry. For small $\lambda^{2}$ it is legitimate to replace the continuous variable $\chi_{\mathbf{r}}$ by a discrete one $\chi_{\mathbf{r}} \approx 2 \pi k_{\mathbf{r}} / q$ leading to

$$
S \approx-\frac{1}{K \lambda^{2}} \sum_{\mathbf{r} \mu} \cos \left(2 \pi \nabla_{\mu} k_{\mathbf{r}} / q\right)
$$

which is just the familiar $q$-state clock model. Equation (16) is exact in the limit $\lambda \rightarrow 0$, where the well known duality between $Z_{q}$-gauge theory and the $q$-state clock model is regained. Equation (13) remains valid for arbitrary $\lambda$.

Note that for fundamental matter, when $q=1$, Eq. (13) essentially describes an $X Y$ model in a finite external magnetic field, which does not have a phase transition; this agrees with Ref. 5 .

\section{Domain wall energy}

We now proceed to show that the domain wall energy in the $q$-state clock symmetric model, Eq. (13), corresponds precisely to the large Wilson loop $\tilde{W}$ in the original compact gauge theory. Consider a finite sized system with side length $L$ and periodic boundary conditions. A domain wall can be forced into the system by changing the boundary conditions such that $\chi\left(\mathbf{r}+L \mathbf{e}_{\nu}\right)=\chi(\mathbf{r})+\Delta \chi, \Delta \chi=2 \pi k / q$. The domain wall energy is defined as the free energy cost $\Delta F$ for introducing such a twist. It is convenient to make a change of variables in the twisted system to get back to periodic boundary conditions by setting $\chi(\mathbf{r})=\theta(\mathbf{r})+\Delta \theta(\mathbf{r})$, where $\theta$ obeys periodic boundary conditions, and $\Delta \theta(\mathbf{r})=$ constant except across a surface $\mathcal{S}$ occupying a cross section of the system perpendicular to the direction $\nu$, where $\Delta \theta(\mathbf{r})$ jumps discontinuously by $2 \pi k / q$. We can then evaluate the domain wall energy as

$$
\Delta F=F_{\text {twisted }}-F_{\text {untwisted }}=-\ln \left\langle e^{-\Delta S}\right\rangle
$$

where the average $\langle\cdots\rangle$ is taken with respect to the unperturbed system, and $\Delta S=S[\theta+\Delta \theta]-S[\theta]$. Going back to the flux line representation Eq. (10) we have

$$
\begin{aligned}
\Delta S & =i \sum_{\mathbf{r}} \Delta \theta_{\mathbf{r}}\left(\nabla \cdot \mathbf{m}_{\mathbf{r}}-q N_{\mathbf{r}}\right) \\
& =-i \sum_{\mathbf{r}} \mathbf{m}_{\mathbf{r}} \cdot \nabla \Delta \theta_{\mathbf{r}}+\Delta \theta_{\mathbf{r}} q N_{\mathbf{r}} \\
& =-\frac{2 \pi i k}{q} \sum_{\mathbf{r} \in \mathcal{S}} \mathbf{m} \cdot \hat{\mathbf{n}}_{\mathbf{r}}-i \sum_{\mathbf{r}} \Delta \theta_{\mathbf{r}} q N_{\mathbf{r}}
\end{aligned}
$$


where $\mathcal{S}$ is the surface at which $\Delta \theta$ is discontinuous and $\hat{\mathbf{n}}_{\mathbf{r}}$ is the surface normal at lattice site $\mathbf{r}$. Because $\Delta \theta_{\mathbf{r}}$ is an integer multiple of $2 \pi / q$ only the first term on the right hand side contribute when exponentiated. The resulting formula is more transparent when written in the continuum,

$$
\left\langle e^{-\Delta S}\right\rangle=\left\langle e^{\frac{2 \pi i k}{q} \int_{\mathcal{S}} \mathbf{m} \cdot d \mathbf{S}}\right\rangle=\left\langle e^{\frac{2 \pi i k}{q} M_{\nu}}\right\rangle .
$$

This is nothing but the large Wilson loop $\tilde{W}$ for a test particle with fractional charge $k$. Thus the domain wall energy of the dual model is related to $\tilde{W}$ by

$$
\Delta F=-\ln \tilde{W} .
$$

In the (dual) ordered phase this will be proportional to the surface area, $\Delta F \sim L^{d-1}=L^{2}$, consistent with an area law for the Wilson loop $\tilde{W} \sim \exp \left(-c L^{2}\right)$. In the disordered phase, creating a domain wall costs no energy and $\Delta F \rightarrow 0$ with system size. Precisely at a critical point $\Delta F \rightarrow$ a universal constant, independent of system size. For a finite sized system we expect the finite size scaling relation

$$
\Delta F(\delta, L)=f\left(\delta L^{1 / \nu}\right)
$$

to hold, where $\delta=\left(K^{-1}-K_{c}^{-1}\right) / K_{c}^{-1}$ is the tuning parameter of the transition and $\nu$ the correlation length exponent.

From simulation data the critical point can thus be located by plotting $\Delta F$ (or equivalently $\tilde{W}$ ) vs the tuning parameter for different system sizes and observing the point where the different curves cross. We will show examples of this below in Fig. 3

\section{Topological order}

One characteristic feature of systems displaying topological order is a ground state degeneracy which depends on the topology of the space on which the model is defined. A further prediction is that for finite sized systems the exact degeneracy will be lifted due to tunneling events, leading to a ground state splitting $\Delta \sim$ $\exp (-L / \xi) \stackrel{16}{16}$ In Ref. 29 it was shown that there is a close relationship between the lifting of the ground state degeneracy and the large Wilson loop $\tilde{W}$ discussed above. Here we give more details on this relation and discuss the generalization to topologies other than the torus.

To simplify the discussion we consider first the case $q=2$ on a torus, with $\tilde{W}$ in the space-time plane. In order to study ground state properties of the system we have to consider anisotropic systems with size $L \times L \times \beta$ and take the limit where the length of the time dimension $\beta$ goes to infinity. In the infinite system size limit the degenerate ground states may be characterized by having an even or odd number of flux quanta $\Phi_{0}$ through the holes of the torus, giving a degeneracy of $2^{2}=4$ since a torus has two holes ${ }^{32}$ The general case of arbitrary $q$

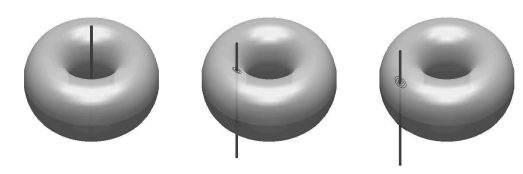

FIG. 2: A flux quantum tunnels out from the hole of a torus.

on a manifold with genus $g$ (i.e., which has $g$ handles and $2 g$ holes) naturally leads to $q^{2 g}$ degenerate ground states. For finite system size the degeneracy will be lifted by vortex tunneling processes, in which a vortex tunnels around a nontrivial path on the torus as illustrated in Fig. 2 In the deconfined phase such processes are exponentially suppressed and the system is topologically ordered. In the confinement phase, however, the vortices condense, and the tunneling is no longer negligible even in the thermodynamic limit. Let us concentrate on just one of the two holes of a torus for now. We will denote the quantum mechanical states with an even or odd number of flux quanta through the hole by $|0\rangle$ and $|1\rangle$, respectively.

The space-time configurations of vortex lines and monopoles that contribute to the partition function splits into different topological sectors, depending on whether the winding number $M_{\nu}$ defined in (6) is even or odd, i.e., $Z=Z_{\text {even }}+Z_{\text {odd }}$. Our Wilson loop $\tilde{W}$ (for $q=2$ ) can be written in terms of these as

$$
\tilde{W}=\left\langle e^{i \pi M_{\nu}}\right\rangle=\frac{Z_{\text {even }}-Z_{\text {odd }}}{Z_{\text {even }}+Z_{\text {odd }}} .
$$

Quantum mechanically the configurations in the even sector contribute to the diagonal matrix elements

$$
Z_{\text {even }}=\left\langle 0\left|e^{-\beta \hat{H}}\right| 0\right\rangle=\left\langle 1\left|e^{-\beta \hat{H}}\right| 1\right\rangle,
$$

while the odd configurations contribute to tunneling matrix elements

$$
Z_{\text {odd }}=\left\langle 0\left|e^{-\beta \hat{H}}\right| 1\right\rangle=\left\langle 1\left|e^{-\beta \hat{H}}\right| 0\right\rangle .
$$

The tunneling lifts the degeneracy and mixes the different flux states. The Hamiltonian restricted to the (almost) degenerate ground state Hilbert space is

$$
\hat{H}=\left(\begin{array}{cc}
E_{0} & -\Delta \\
-\Delta & E_{0}
\end{array}\right)
$$

which has energy eigenvalues $E=E_{0} \mp \Delta$ and eigenstates $| \pm\rangle=(|0\rangle \pm|1\rangle) / \sqrt{2}$. The ground state splitting $\Delta$ can be related to $\tilde{W}$ by the following equality, valid for $\beta \rightarrow$ $\infty$ :

$e^{-\beta \hat{H}}=e^{-\beta E_{0}}\left(\begin{array}{cc}\cosh \beta \Delta & \sinh \beta \Delta \\ \sinh \beta \Delta & \cosh \beta \Delta\end{array}\right)=\left(\begin{array}{cc}Z_{\text {even }} & Z_{\text {odd }} \\ Z_{\text {odd }} & Z_{\text {even }}\end{array}\right)$.

Using Eq. 221) we have

$$
\tilde{W}=e^{-2 \beta \Delta},
$$


as found in $\operatorname{Ref} 29$. In the deconfined phase $\tilde{W}=1$ in the thermodynamic limit and we must have $\Delta=0$ for $\beta \rightarrow$ $\infty$, i.e., an exact degeneracy. For finite $L$ the splitting can be evaluated directly deep in the deconfinement phase, where the flux lines cost much energy and are very dilute. The dominating configurations which have to be taken into account then are those with $n$ straight tunneling trajectories across the system with action $S \approx \sigma L n$. This gives

$$
Z \approx \sum_{n} \frac{1}{n !}(\beta L)^{n} e^{-\sigma L n}=e^{\beta L e^{-\sigma L}}
$$

and

$$
\tilde{W} Z \approx \sum_{n} \frac{(-1)^{n}}{n !}(\beta L)^{n} e^{-\sigma L n}=e^{-\beta L e^{-\sigma L}},
$$

where $\sigma$ is the line tension and there are $\beta L$ places to put the tunneling trajectory. In this limit $\tilde{W}$ will thus be given by $\tilde{W} \approx \exp \left(-2 \beta L e^{-\sigma L}\right)$. Precisely such an exponential form of the ground state splitting has been argued for by Wen ${ }^{33}$ Approaching the phase transition the flux line will no longer be straight and interaction effects between different flux lines must be taken into account, making it much more difficult to estimate the ground state splitting. However, matching to the scaling form Eq. (21) for $\tilde{W}$ suggests that $\tilde{W}=\exp \left(-2 \beta L / \xi^{2} e^{-\sigma L / \xi}\right)$, so that

$$
\Delta=L / \xi^{2} e^{-\sigma L / \xi},
$$

where $\xi \sim|\delta|^{-\nu}$ is the correlation length. This should hold for $L \gtrsim \xi$ on the deconfined side of the transition. Indeed, we will verify in the next sections that this form is in agreement with the data from numerical simulations. In the confinement phase an area law $\tilde{W} \sim e^{-c \beta L}$ for the Wilson loop translates into a splitting $\Delta \sim L$ that grows with system size.

The arguments given above naturally generalize to manifolds of arbitrary genus $g$ and charge $q$. There are $2 g$ nontrivial loops around which vortex tunneling can take place. Each such tunneling event changes the flux through the corresponding hole by one flux quantum $\Phi_{0}$. In the infinite system size limit the $q^{2 g}$ flux states $|\vec{f}\rangle=\left|f_{1}\right\rangle\left|f_{2}\right\rangle \ldots\left|f_{2 g}\right\rangle$, where $f_{i} \in \mathbb{Z}_{q}$ is the number of flux quanta through hole $i(\bmod q)$, are degenerate ground states. The tunneling lifts the degeneracy leading to a splitting

$$
\Delta E_{\vec{k}}=-\frac{1}{\beta} \ln \tilde{W}_{\vec{k}}
$$

from the ground state. Here we generalize Eq. (9) to

$$
\tilde{W}_{\vec{k}}=\left\langle\exp \left(\frac{2 \pi i}{q} \sum_{i=1}^{2 g} k_{i} M_{i}\right)\right\rangle,
$$

where $M_{i}$ measures the flux quanta $(\bmod q)$ tunneling through the space-time surface surrounding hole $i$, and $k_{i} \in \mathbb{Z}_{q}$. The corresponding eigenstates are given in the appendix in Eq. A.2 and the ground state is the product of the symmetric combinations of all the flux states

$$
|G\rangle=\prod_{i=1}^{2 g}\left(\frac{1}{\sqrt{q}} \sum_{f_{i}=0}^{q-1}|f\rangle_{i}\right) .
$$

Away from the transition one may, to a very good approximation, neglect higher order tunneling processes and only consider those which tunnel single flux quanta. Denoting those tunneling matrix elements by $T_{i}$ the energy levels become $E_{\vec{k}}=E_{0}-\sum_{i} T_{i} 2 \cos \left(2 \pi k_{i} / q\right)$, i.e., the contributions from individual tunneling processes are just added together.

\section{E. Vortex gap}

An important property of the ordered phase is that vortex excitations are gapped. This gap can be related to the large Wilson loop oriented normal to the time direction $\tilde{W}_{\tau}$. Returning to the $q=2$ case the vortex gap is

$$
E_{v}=-\lim _{\beta \rightarrow \infty} \frac{1}{\beta} \ln \frac{Z_{\text {odd }}}{Z_{\text {even }}}=-\lim _{\beta \rightarrow \infty} \frac{1}{\beta} \ln \frac{1-\tilde{W}_{\tau}}{1+\tilde{W}_{\tau}},
$$

where $Z_{\text {odd }}$ and $Z_{\text {even }}$ now refer to the partition functions with an odd and even number of vortices present. A scaling argument analogous to Eq. (30) gives

$$
\xi E_{v}=\sigma+\frac{2 \xi}{\beta} \ln \frac{L}{\xi},
$$

on the deconfined side of the transition in the low temperature limit $\beta \rightarrow \infty$ and $\xi \lesssim L$.

\section{MONTE CARLO ALGORITHM}

We use Monte Carlo simulations to obtain quantitative results for the model and the nonlocal order parameter $\tilde{W}$. The simulations are performed in the loop-gas representation, Eq. (3), of flux lines and monopoles, and we focus on the cases $q=2$ and $q=3$. We use simple cubic lattices with periodic boundary conditions for the vortices and monopoles, corresponding to fluctuating twist boundary conditions in the phase representation ${ }^{29}$

In the simulation we must update both the flux lines and the monopoles. In order to calculate $\tilde{W}$ it is necessary to include global moves that change the winding numbers $M_{\nu}$ and thus the topology of the vortex configuration. In a conventional Metropolis algorithm the acceptance ratio for such moves becomes exponentially small with increased lattice size. To overcome this we use a worm cluster Monte Carlo algorithm ${ }^{34.35}$ This algorithm is described for on-site interactions in Ref. 34, but is straightforward to adapt to longer range interactions. 

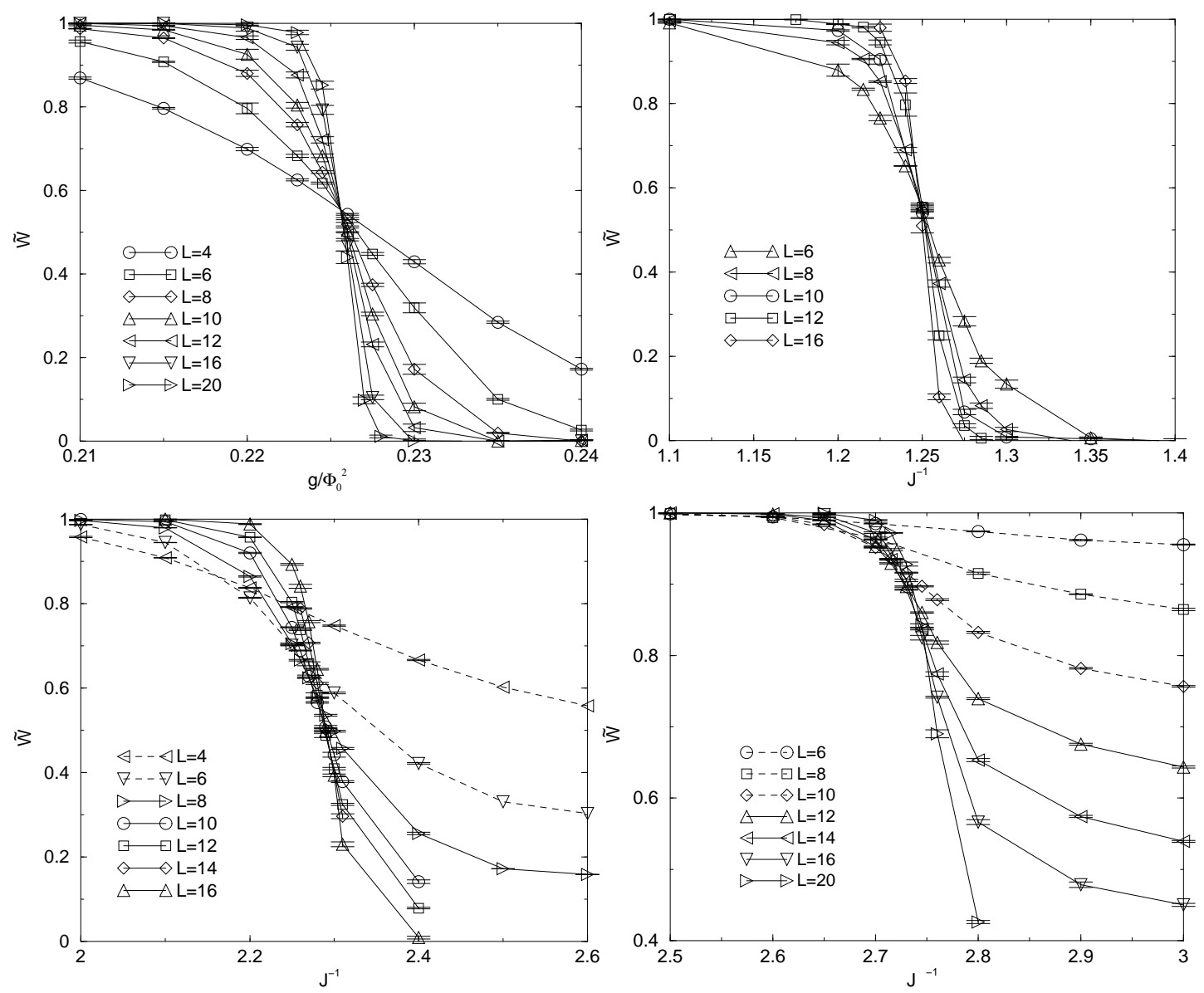

FIG. 3: The large Wilson loop order parameter $\tilde{W}$ as function of coupling constant, for different values of the screening length $\lambda$. Upper left: $\tilde{W}$ vs $g / \Phi_{0}^{2}$ for $\lambda=0$. Upper right, lower left, and lower right: $\tilde{W}$ vs $J^{-1}$ for $\lambda=0.5,1,2$, respectively. The critical point is where the curves for different system sizes cross. Note that only system sizes much larger than $\lambda$ scale well (solid lines), smaller sizes (dashed lines) suffers from finite size corrections.

To construct a worm one starts at a random lattice site $s_{1}$ in space-time. A vortex segment is then created in direction $\sigma$ to a new lattice site $s_{2}$. The direction is chosen with the probability

$$
P_{\sigma} \sim A_{\sigma}=\min \left(1, \exp \left(-\Delta S_{\sigma}\right)\right),
$$

where $\Delta S_{\sigma}$ is the change in the action $S$ for creating a vortex segment in direction $\sigma$. A new direction and lattice site are then chosen from $s_{2}$. These steps are repeated until the worm of vortex segments form a closed loop. To ensure detailed balance, the final worm is accepted with the probability $P=$ $\min \left[1, N\right.$ (noworm) $/ N$ (worm)], where $N=\sum_{\sigma} A_{\sigma}$ at the initial site, before and after the creation of the loop. Occasionally the loop constructed this way winds around the system, leading to the desired changes in the winding number $M_{\nu}$.

The model allows magnetic monopoles in addition to the flux lines. The monopoles are updated using a standard Metropolis scheme, with trial moves consisting of the insertion of a nearest neighbor pair of oppositely charged monopoles connected by $q$ flux quanta on the link between them. These moves are then accepted with probability $\min (1, \exp (-\Delta S))$. The creation of a plus monopole on top of a minus monopole (or vice versa) leads to their annihilation so that the procedure described includes creation, destruction, as well as motion of monopoles.

It is straightforward to show that the algorithm described above, combining both the worm updates of the flux lines and the Metropolis monopole moves, fulfills both ergodicity and detailed balance, and respects the constraint $\nabla \cdot \mathbf{m}=q N$. The Wilson loop order parameter $\tilde{W}_{\mu}$ in direction $\mu$ is calculated in the simulations as $\left\langle\cos \left(2 \pi M_{\mu} / q\right)\right\rangle$ where $M_{\mu}$ is the number of vortex lines crossing a plane, perpendicular to direction $\mu$, of the system. 


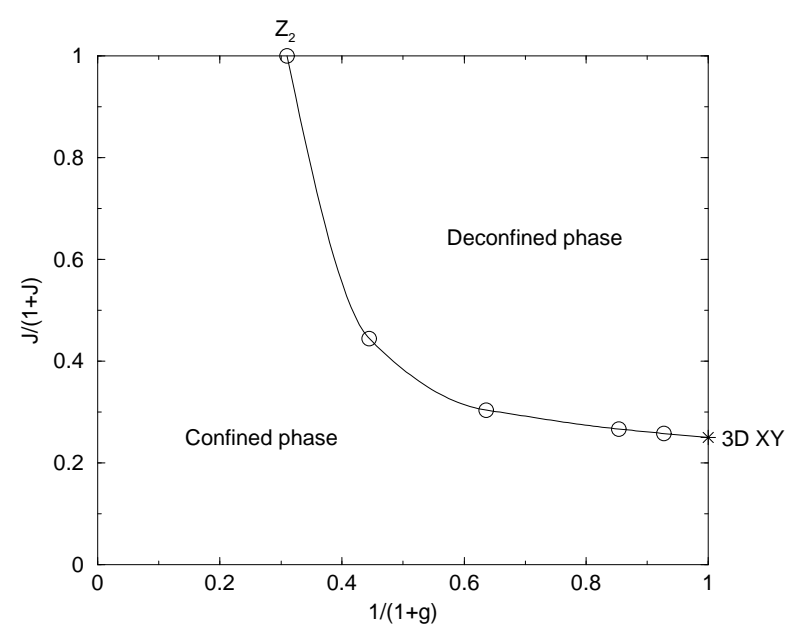

FIG. 4: Phase diagram of the model with $q=2$. Circles indicate the points which have been simulated. The parameters have been rescaled in order to fit the phase diagram into the figure, so that $J \rightarrow \infty$ occur at the upper side and $g \rightarrow 0$ at the right side. The phase diagram connects the $3 \mathrm{D} X Y$ point at $g=0$ and the $Z_{2}$ transition at strong coupling $J \rightarrow \infty$. The deconfined phase is topologically ordered.

\section{RESULTS}

For $q=2$ we have made simulations for constant $\lambda=0,0.5,1.0,2.0$, varying $J$ (or $g$ in the $\lambda=0$ case) in each simulation. The results for $\tilde{W}$ are shown in Fig. 3 Below the transition $\tilde{W}$ goes to one with increasing system size. This is the deconfinement phase. Above the transition, in the confinement phase, it goes to zero. Precisely at the transition $\tilde{W}$ should be independent of system size according to the scaling formula Eq. (21). The figures clearly show how $\tilde{W}$ for system sizes much larger than $\lambda$ cross at a given $J_{c}$. However, it is also apparent that for large values of $\lambda$ there are strong corrections to scaling which influence the smaller sizes. This is not surprising since the interaction Eq. (4) looks screened only at distances much larger than $\lambda$. It is important to have this in mind during the finite size scaling analysis below. The phase diagram determined from the simulations is shown in Fig. 4

As discussed $\tilde{W}$ differs from an ordinary Wilson loop in that it does not follow a perimeter law in the deconfined phase. In order to demonstrate both area and perimeter law, we use a Wilson loop that covers one quarter of the system. This configuration is less suitable to locate $J_{c}$, but demonstrates the area and perimeter laws nicely in Fig. 5 .

We also simulate the model with $q=3$ to show that $\tilde{W}$ works also for larger values of $q$. The result for $\tilde{W}$ is shown in Fig. 7 for $\lambda=0$. For $q=3$ the model has a first order transition when $\lambda=0, \underline{28}$ and hence we expect no scaling of $\tilde{W}$. This is consistent with our MC data, since we find no good crossing. Instead the transition becomes sharper with increasing system size and it is conceivable that a discontinuity develops in the thermodynamic limit.

We now turn to the finite size scaling relation given in Eq. (21), and the correlation length exponent $\nu$, for $q=2$. With an appropriate choice of $\nu$ the data for different sizes should collapse onto a single scaling function. This is achieved by minimizing

$$
\sum_{L} \int\left[\tilde{W}_{L}(x)-\overline{\tilde{W}}(x)\right]^{2} d x
$$

where $x=\left(J^{-1}-J_{c}^{-1}\right) L^{1 / \nu}$ and $\bar{W}$ is the mean value of $\tilde{W}_{L}$. We calculate $\nu$ and $J_{c}$ by making a multi parameter minimization of Eq. (37). Figure [6 shows two of the resulting data collapses. In doing this minimization it is important to make use only of system sizes large enough that corrections to scaling are negligible, hence we include only those sizes for which well defined crossings were found above. We get $\nu=0.633 \pm 0.007$, $0.64 \pm 0.015,0.62 \pm 0.03,0.6 \pm 0.15$, for $\lambda=0,0.5,1,2$, respectively. Within error-bars the data for all values of $\lambda$ that we have tested are consistent with the expected $\nu \approx 0.63$ of the 3D Ising model, which is the dual of the $Z_{2}$ gauge theory, i.e., the limiting case obtained for $\lambda \rightarrow 0$. This is in sharp contrast to the continuously varying exponents found by Sudbø et al..$^{27}$ and Smiseth et $a l \stackrel{28}{2}$ Their results were obtained from studies of the third moment of the action and showed substantial deviations from the $3 \mathrm{D}$ Ising value for $\lambda \gtrsim 0.5$. The parameter values used in these papers overlap to large extent with the present ones, although there is a slight shift in the location of the phase diagram resulting from our use of the Villain model [Eq. (2), or equivalently (3)] instead of the cosine version Eq. (11). This is not expected to change the critical exponents, however.

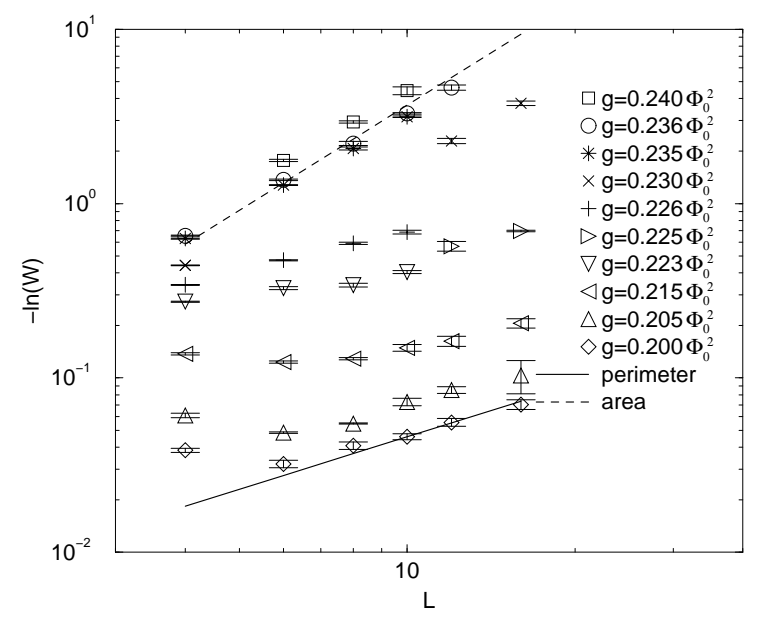

FIG. 5: Area law and perimeter law demonstrated for $\lambda \rightarrow 0$, for a Wilson loop $W(\mathcal{C})$ which covers a quarter of a cross section of the system and therefore has a finite perimeter and area. Dashed and solid lines are exact area and perimeter laws, inserted for comparison. 

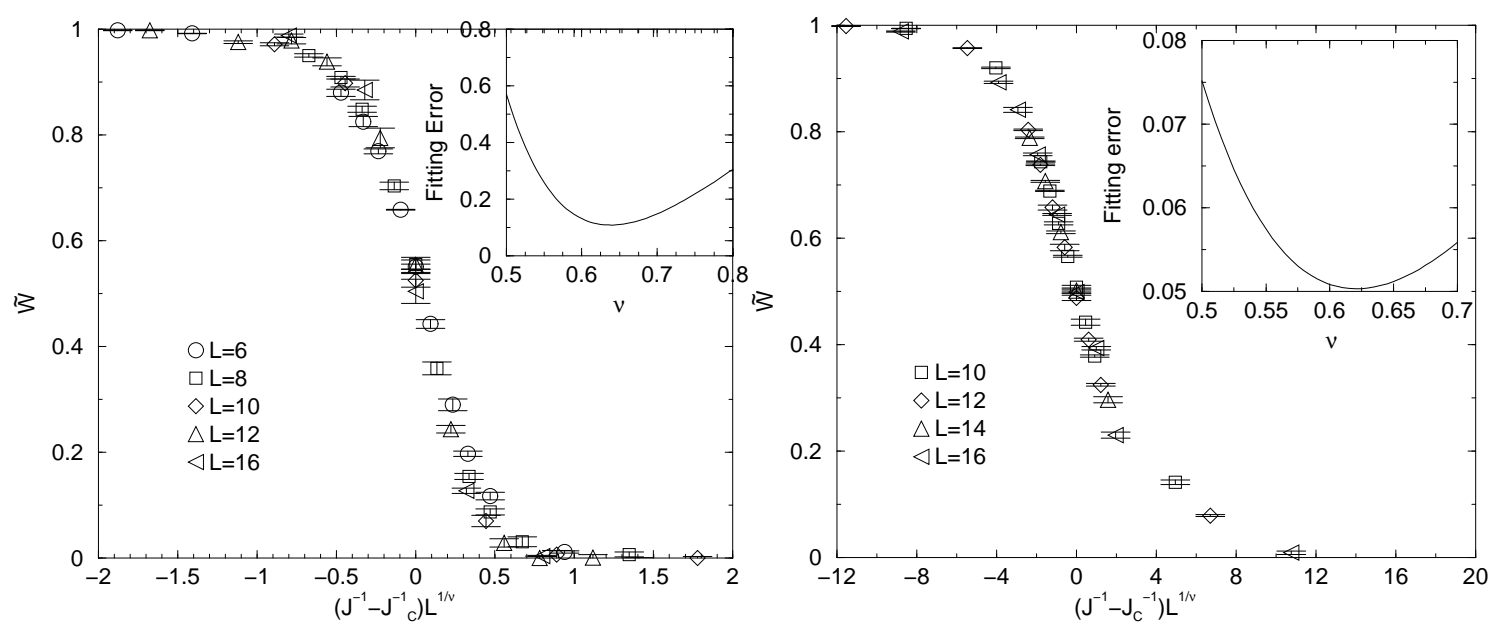

FIG. 6: Finite size scaling plot of the $\tilde{W}$ order parameter for $\lambda=0.5$ (left) with $\nu=0.64$ and $\lambda=1$ (right) with $\nu=0.62$. $J_{c}^{-1}$ is determined from Fig. 3 to 1.25 and 2.29, respectively. The inset shows fitting error as a function of $\nu$.

Finally, we may also use $\tilde{W}$ to calculate two quantities which are characteristic of topological order, namely the gap to vortex excitations $E_{v}$, and the splitting $\Delta$ of the nearly degenerate ground states on a torus. Figure 8 shows $\xi E_{v}$ calculated using Eq. (34) as a function of $\xi / \beta$, where $\xi \sim|\delta|^{-\nu}$. The data nicely follows the scaling relation proposed in Eq. (35) in the deconfined phase, with $\sigma \approx 10$.

The relation between $\tilde{W}$ and $\Delta$ in Eq. (27) allows the ground state splitting to be explicitly calculated using the Monte Carlo data. An example of this is shown in Fig. 9 where the exponential dependence on system size obtained in Eq. (30) is clearly seen in the deconfinement phase (solid line), while an area law (dashed line) is found in the confinement phase. For further examples and discussion of this aspect see Ref. 29.

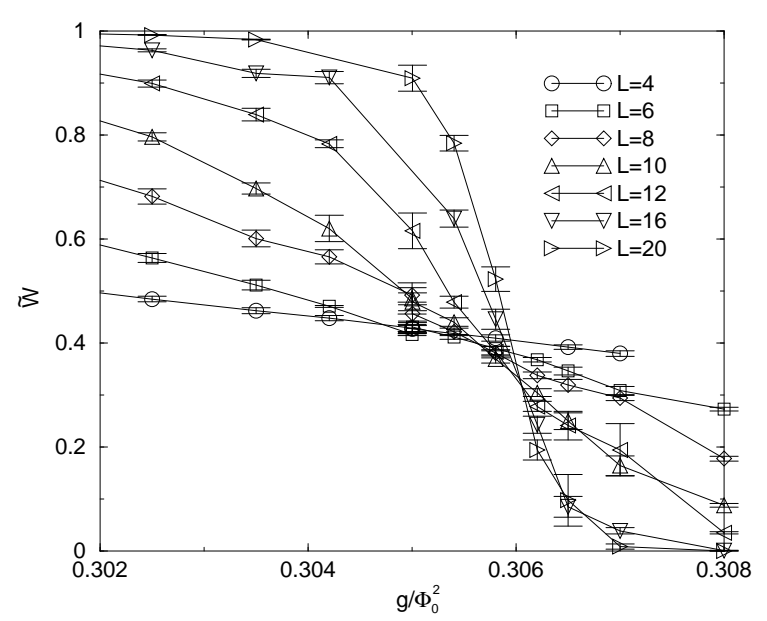

FIG. 7: $\quad \tilde{W}$ vs $g / \Phi_{0}^{2}$ for a model with $q=3$ and $\lambda=0$. Note that this model has a first order transition and no scaling behavior is expected.

\section{SUMMARY}

In summary, we have studied a compact $U(1)$ gauge theory coupled to bosonic matter with gauge charge $q$ using duality arguments and Monte Carlo simulations. The confinement-deconfinement transition is analyzed using a nonlocal order parameter $\tilde{W}$, which is related to large Wilson loops with the loop covering a whole cross section of the system, and which directly probes the confining properties of the theory. The model can be mapped to a dual model with a global $q$-state clock symmetry, and $\tilde{W}$ corresponds to the domain wall energy of that model, which suggests a scaling behavior according to Eq. (21). We confirm this using Monte Carlo simulations and study the details of the deconfinement transition over a range of parameters. Our results for the critical exponent $\nu$ support a transition in the 3D Ising universality class for

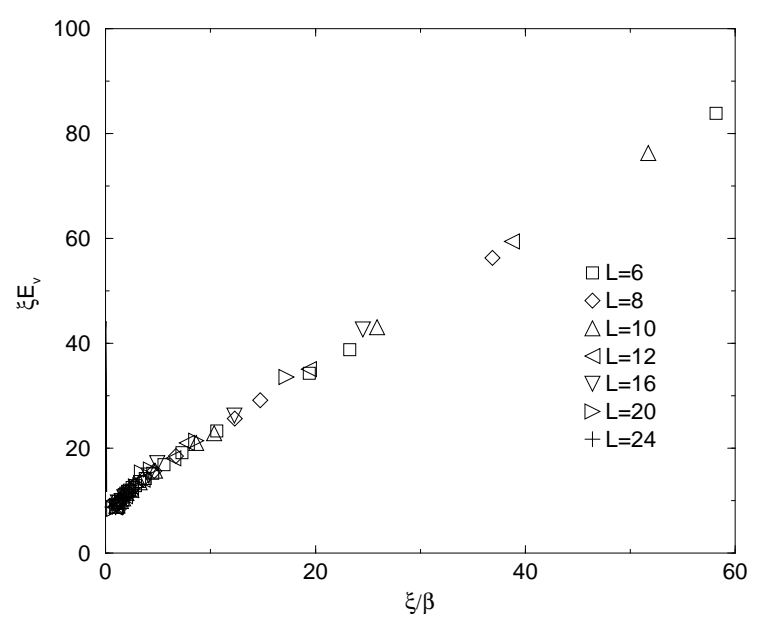

FIG. 8: The energy gap to vortex excitations as a function of temperature in the deconfined phase, for $q=2$ and $\lambda=0$. 


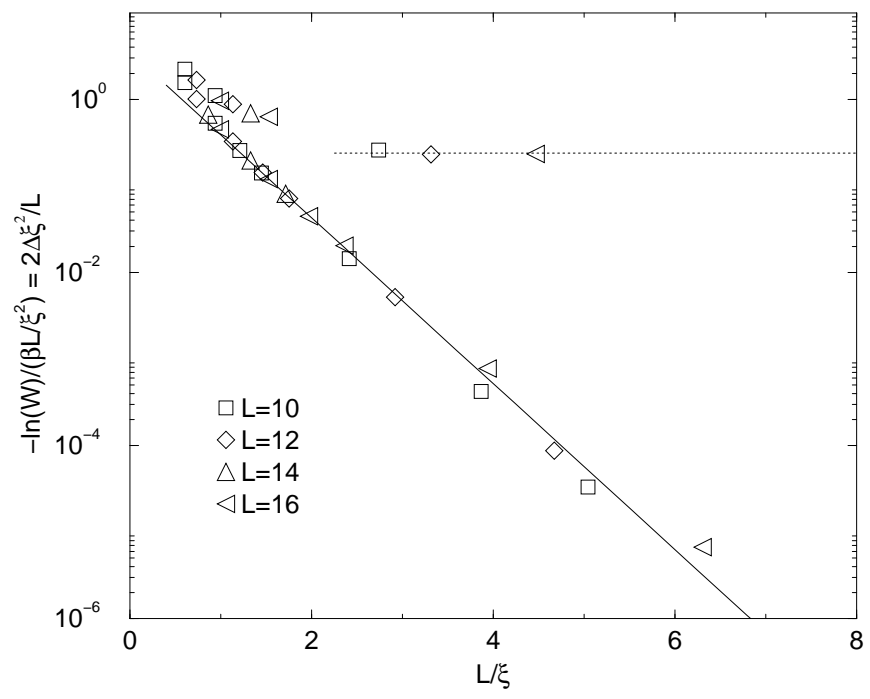

FIG. 9: The scaling combination $2 \Delta \xi^{2} / L$ plotted against $L / \xi$ for $q=2, \lambda=1$. In the topologically ordered deconfinement phase the data follows the exponential dependence given in Eq. (30), as indicated by the solid line. The dashed line indicates the area law obeyed in the confinement phase.

all values of $\lambda$ tested. In particular, we do not see any trace of continuously varying critical exponents found in Refs. 27 28 using a different order parameter. There are, however, strong crossover effects in the vicinity of the 3D $X Y$ transition at $\lambda \rightarrow \infty$, which make an accurate determination of the exponents tricky in this limit. The deconfined phase is topologically ordered with gapped vortex excitations, with a gap $E_{v} \sim \xi^{-1}$, which can be related to $\tilde{W}$ for a spatial loop. The ground state degeneracy characteristic of topological order is lifted in finite sized systems with a gap $2 \Delta$ which is simply related to $\tilde{W}$ for a space-time loop ${ }^{29}$ This establishes a firm relationship between topological order and deconfinement and allows explicit calculations of the ground state energy splitting in the topologically ordered phase of the theory. We expect the methods developed here to be useful for quantitative studies of topological order in other systems as well.

\section{Acknowledgments}

We thank T. Hans Hanson, and Asle Sudbø for valuable discussions. Support from the Swedish Research
Council (VR) and the Göran Gustafsson foundation is gratefully acknowledged.

\section{APPENDIX: GROUND STATE SPLITTING FOR GENERAL $g$ AND $q$}

In this appendix we give a detailed derivation of the relation between the ground state splitting and $\tilde{W}$ for models with gauge charge $q$ on manifolds with arbitrary genus $g$. The configurations contributing to the partition function can be split into separate topological sectors depending on the flux $\Phi_{0} m_{i}$ passing through a space-time cross section surrounding hole $i$. Let $Z_{\vec{m}}=\left\langle\vec{f}\left|e^{-\beta H}\right| \vec{f}+\vec{m}\right\rangle$ denote the partition function restricted to the sector labeled by $\vec{m}$. Then $\tilde{W}_{\vec{k}}=\sum_{\vec{m}} Z_{\vec{m}} e^{2 \pi i \vec{k} \cdot \vec{m} / q} / \sum_{\vec{m}} Z_{\vec{m}}$. The Hamiltonian in the ground state subspace can be written as

$$
\mathcal{H}=\sum_{\vec{m}} h_{\vec{m}} \sum_{\vec{f}}|\vec{f}\rangle\langle\vec{f}+\vec{m}|+| \vec{f}+\vec{m}\rangle\langle\vec{f}|,
$$

where $h_{\overrightarrow{0}}=E_{0} / 2$ and $h_{\vec{m}}=T_{\vec{m}}(\vec{m} \neq \overrightarrow{0})$ are tunneling amplitudes. Because of translation invariance in the index $\vec{f}$ the eigenstates are

$$
|\tilde{k}\rangle=\frac{1}{\sqrt{q^{2 g}}} \sum_{\vec{f}} e^{2 \pi i \vec{k} \cdot \vec{f} / q}|\vec{f}\rangle
$$

with corresponding eigenvalues

$$
E_{\vec{k}}=\sum_{\vec{m}} h_{\vec{m}} 2 \cos (2 \pi \vec{k} \cdot \vec{m} / q) .
$$

To relate the energies to the $\tilde{W}_{\vec{k}}$ that are easily calculated in a simulation we use

$$
\begin{aligned}
e^{-\beta E_{\vec{k}}}= & \left\langle\tilde{k}\left|e^{-\beta \mathcal{H}}\right| \tilde{k}\right\rangle= \\
& \frac{1}{q^{2 g}} \sum_{\vec{f}, \vec{f}^{\prime}}\left\langle\overrightarrow{f^{\prime}}\left|e^{-\beta \mathcal{H}}\right| \vec{f}\right\rangle e^{2 \pi i \vec{k} \cdot\left(\vec{f}-\vec{f}^{\prime}\right) / q}= \\
& \sum_{\vec{m}} Z_{\vec{m}} e^{2 \pi i \vec{k} \cdot \vec{m} / q}=\tilde{W}_{\vec{k}} \sum_{\vec{m}} Z_{\vec{m}},
\end{aligned}
$$

from which Eq. (31) follows.
* Electronic address: anders@condmat.physics.kth.se

$\dagger$ Electronic address: jlidmar@kth.se

1 G. Baskaran and P. W. Anderson, Phys. Rev. B 37, R580 (1988).

2 L. B. Ioffe and A. I. Larkin, Phys. Rev. B 39, 8988 (1989).

3 N. Nagaosa and P. A. Lee, Phys. Rev. B 45, 966 (1992).
4 P. A. Lee and N. Nagaosa, Phys. Rev. B 46, 5621 (1992).

${ }^{5}$ N. Nagaosa and P. A. Lee, Phys. Rev. B 61, 9166 (2000).

${ }^{6}$ N. Read and S. Sachdev, Phys. Rev. Lett. 66, 1773 (1991).

7 S. Sachdev and N. Read, Int. J. Mod. Phys. B 5, 219 (1991).

8 S. Sachdev, Rev. Mod. Phys. 75, 913 (2003). 
${ }^{9}$ X.-G. Wen and P. A. Lee, Phys. Rev. Lett. 76, 503 (1996).

10 T. Senthil and M. P. A. Fisher, Phys. Rev. B 63, 134521 (2001).

11 C. Mudry and E. Fradkin, Phys. Rev. B 49, 5200 (1994).

12 C. Mudry and E. Fradkin, Phys. Rev. B 50, 11409 (1994).

13 D.-H. Lee, Phys. Rev. Lett. 84, 2694 (2000).

14 I. Ichinose, T. Matsui, and M. Onoda, Phys. Rev. B 64, 104516 (2001).

15 T. Senthil and O. Motrunich, Phys. Rev. B 66, 205104 (2002).

16 X.-G. Wen, Int. Journ. Mod. Phys. B 5, 1641 (1991).

17 L. B. Ioffe, M. V. Feigel'man, A. Ioselevich, D. Ivanov, M. Troyer, and G. Blatter, Nature 415, 503 (2002).

18 A. Y. Kitaev, Ann. Phys. 303, 2 (2003).

19 M. Franz and Z. Tesanovic, Phys. Rev. Lett. 87, 257003 (2001).

20 I. F. Herbut, Phys. Rev. B 66, 094504 (2002).

${ }^{21}$ I. F. Herbut and B. H. Seradjeh, Phys. Rev. Lett. 91, 171601 (2003).

${ }^{22}$ M. Hermele, T. Senthil, M. P. A. Fisher, P. A. Lee, N. Nagaosa, and X.-G. Wen, Phys. Rev. B 70, 214437 (2004).

23 H. Kleinert, F. S. Nogueira, and A. Sudbø, Phys. Rev. Lett. 88, 232001 (2002).

24 A. M. Polyakov, Nucl. Phys. 120, 429 (1977).

25 E. Fradkin and S. H. Shenker, Phys. Rev. D 19, 3682
(1979).

26 X. G. Wen, Phys. Rev. B 44, 2664 (1991).

27 A. Sudbø, E. Smørgrav, J. Smiseth, F. S. Nogueira, and J. Hove, Phys. Rev. Lett. 89, 226403 (2002).

28 J. Smiseth, E. Smørgrav, F. S. Nogueira, J. Hove, and A. Sudbø, Phys. Rev. B 67, 205104 (2003).

29 A. Vestergren, J. Lidmar, and T. H. Hansson, Europhys. Lett. 69, 256 (2005).

30 M. B. Einhorn and R. Savit, Phys. Rev. D 17, 2583 (1978).

31 M. N. Chernodub, R. Feldmann, E.-M. Ilgenfritz, and A. Schiller, Phys. Lett. B 605, 161 (2005), arXiv:heplat/0502009.

32 T. H. Hansson, V. Oganesyan, and S. L. Sondhi, Ann. Phys. 313, 497 (2004).

33 X.-G. Wen and Q. Niu, Phys. Rev. B 41, 9377 (1990).

${ }^{34}$ F. Alet and E. S. Sørensen, Phys. Rev. E 67, 015701(R) (2003).

35 N. V. Prokof'ev, B. V. Svistunov, and I. S. Tupitsyn, Phys. Lett. A 238, 253 (1998).

36 Strictly speaking the flux lines and monopoles live on the dual lattice of the original model. Since this is also a cubic lattice, shifted by half a lattice constant in the (111) direction we use the same index $\mathbf{r}$ to denote sites on this dual lattice. 